\title{
Philosophiques
}

\section{Preuves par excellence}

\section{Jacques Dubucs et Sandra Lapointe}

Volume 30, numéro 1, printemps 2003

Bernard Bolzano. Philosophie de la logique et théorie de la connaissance

URI : https://id.erudit.org/iderudit/007740ar

DOI : https://doi.org/10.7202/007740ar

Aller au sommaire du numéro

\section{Éditeur(s)}

Société de philosophie du Québec

ISSN

0316-2923 (imprimé)

1492-1391 (numérique)

Découvrir la revue

Citer cet article

Dubucs, J. \& Lapointe, S. (2003). Preuves par excellence. Philosophiques, 30(1), 219-234. https://doi.org/10.7202/007740ar

\section{Résumé de l'article}

Bolzano fut le premier philosophe à établir une distinction explicite entre les procédés déductifs qui nous permettent de parvenir à la certitude d'une vérité et ceux qui fournissent son fondement objectif. La conception que Bolzano se fait du rapport entre ce que nous appelons ici, d'une part, « conséquence subjective ", à savoir la relation de raison à conséquence épistémique et, d'autre part, la " conséquence objective ", c'est-à-dire la fondation (Abfolge), suggère toutefois que Bolzano défendait une conception " explicativiste " de la preuve : les preuves par excellence sont celles qui reflètent l'ordre de la fondation objective. Dans cet article nous faisons état des problèmes liés à une telle conception et argumentons en faveur d'une démarcation plus stricte entre la préoccupation ontologique et la préoccupation épistémologique dans l'élaboration d'une théorie de la preuve.
Ce document est protégé par la loi sur le droit d'auteur. L'utilisation des services d’Érudit (y compris la reproduction) est assujettie à sa politique d'utilisation que vous pouvez consulter en ligne.

https://apropos.erudit.org/fr/usagers/politique-dutilisation/ 


\title{
Preuves par excellence
}

\author{
JACQUES DUBUCS \\ CNRS/IHPST-Paris \\ jacques.dubucs@cnrs-dir.fr
}

\section{SANDRA LAPOINTE}

Université du Québec à Montréal

lapointe.sandra@sympatico.ca

\begin{abstract}
RÉSUMÉ. - Bolzano fut le premier philosophe à établir une distinction explicite entre les procédés déductifs qui nous permettent de parvenir à la certitude d'une vérité et ceux qui fournissent son fondement objectif. La conception que Bolzano se fait du rapport entre ce que nous appelons ici, d'une part, «conséquence subjective», à savoir la relation de raison à conséquence épistémique et, d'autre part, la «conséquence objective», c'est-à-dire la fondation (Abfolge), suggère toutefois que Bolzano défendait une conception «explicativiste» de la preuve : les preuves par excellence sont celles qui reflètent l'ordre de la fondation objective. Dans cet article nous faisons état des problèmes liés à une telle conception et argumentons en faveur d'une démarcation plus stricte entre la préoccupation ontologique et la préoccupation épistémologique dans l'élaboration d'une théorie de la preuve.
\end{abstract}

ABSTRACT. - Bolzano was the first to establish an explicit distinction between the deductive methods that allow us to recognise the certainty of a given truth and those that provide its objective ground. His conception of the relation between what we, in this paper, call "subjective consequence", i.e. the relation from epistemic reason to consequence and "objective consequence", i.e. grounding (Abfolge) however suggests that Bolzano advocated an "explicativist" conception of proof: proofs par excellence are those that reflect the objective order of grounding. In this paper, we expose the problems involved by such a conception and argue in favour of a more rigorous demarcation between the ontological and the epistemological concern in the elaboration of a theory of proof.

1.

Deux contraintes s'exercent sur la notion de preuve. La première est la fiabilité : s'il existe une preuve de $\phi$, alors $\phi$ doit être le cas. La seconde est l'immanence épistémique : s'il existe une preuve de $\phi$, alors cette existence doit pouvoir être reconnue. Une manière de lier les deux contraintes est de dire que la preuve nous assure de la vérité de ce qu'elle prouve comme le papier de tournesol nous assure de l'alcalinité de la solution dans laquelle on l'a plongé : la preuve est ce qui nous permet de détecter la vérité du théorème, ce qui signifie à la fois qu'elle ne peut exister sans qu'il soit vrai et que c'est à son existence que nous reconnaissons qu'il l'est. D'après cette conception «détectrice », la preuve est donc une relation entre un sujet et une proposition, relation qui, lorsqu'elle a lieu, entraîne à la fois la conviction du sujet et la vérité de la proposition. La difficulté de cette conception est que le mot "entraîne ", dans cette phrase, est parfaitement équivoque. Ce qui entraîne la conviction que $\phi$, 
c'est-à-dire, selon cette explication, la preuve, n'est pas ce qui entraîne $\phi$. Je sais que la solution est alcaline parce que je constate que le papier de tournesol a viré de couleur, mais le papier de tournesol a viré parce que la solution est alcaline. Dans cet exemple, comme dans bien d'autres, la conviction que $\phi$ découle de la saisie d'une conséquence de $\phi$ plus facile à saisir que $\phi$ lui-même. Mais ni la saisie de cette conséquence, ni, bien entendu, cette conséquence elle-même, ne peuvent être considérése comme la raison de $\phi$. Si ce qui entraîne la chose prouvée ne peut être identifié à ce qui entraîne la conviction dans la chose prouvée, on a tort de penser que ce qu'on appelle ici indifféremment preuve puisse remplir les deux rôles. Parce que c'est précisément ce que prétend la conception «détectrice» de la preuve, elle est donc absurde.

Bolzano, à qui l'on doit une réfutation de ce genre, propose de distinguer entre deux notions de preuve, c'est-à-dire deux relations de "conséquence » : l'une objective, la fondation (ou relation de fondement à conséquence), l'autre subjective, la relation de raison à conséquence épistémique. De la sorte, il est en mesure de poser une distinction claire entre le fondement objectif (objektiver Grund) d'une vérité et les moyens subjectifs qui nous permettent de la connaître. Si une proposition dépend épistémiquement d'autres propositions, c'est-à-dire si la reconnaissance de sa vérité demande que la vérité de ces autres propositions soit préalablement reconnue, alors elle est une conséquence subjective (subjektive Folge) de ces dernières. Si, par contre, elle dépend aléthiquement d'autres propositions, c'est-à-dire si sa vérité même suppose que ces autres propositions soient vraies, alors elle est une conséquence objective (objektive Folge) de ces dernières. Pour reprendre l'exemple canonique de Bolzano $^{1}$, la proposition que le mercure monte plus haut en été qu'en hiver dans le thermomètre est une conséquence objective de la proposition qu'il fait plus chaud en été qu'en hiver, mais la seconde proposition est une conséquence subjective de la première, puisque c'est en reconnaissant la vérité de la proposition relative au thermomètre que nous reconnaissons celle de la proposition relative à la température. Généralisons sur cet exemple de Bolzano, et supposons que $\psi_{1}, \ldots, \psi n$ soient des conséquences objectives de $\phi$. Alors $\phi$ est conséquence subjective de certaines des propositions $\psi_{i}$, à savoir celles dont la reconnaissance comme vraies mène à la reconnaissance de $\phi$ comme vraie. En somme, la relation de conséquence subjective apparaît donc comme une partie de la converse de la relation de conséquence objective :

\section{(1) $S F \subset O F^{\prime}$}

Naturellement, cette détermination est encore trop vague, car elle n'indique pas quelles sont, parmi les conséquences objectives de $\phi$, celles dont $\phi$ découle subjectivement. De toute évidence, la reconnaissance de la vérité de $\phi$ à partir de la reconnaissance de la vérité de l'une de ses conséquences objectives requiert que soit également reconnu le fait que la vérité de la proposition- 
conséquence (dans notre cas, la hausse du mercure dans le thermomètre) suppose nécessairement la vérité de la proposition $\phi$ (relative à la hausse de la température). Parmi toutes les propositions qui sont conséquences objectives d'une proposition donnée $\phi$, seules ont donc cette proposition comme conséquence subjective les propositions dont nous sommes capables de reconnaître à la fois qu'elles sont vraies et qu'elles ne peuvent l'être sans que $\phi$ le soit aussi. Autrement dit, la relation de conséquence subjective diffère de la relation de conséquence objective sur le point suivant : la relation de conséquence objective est une relation directe entre propositions, alors que la relation de conséquence subjective est une relation entre propositions qui est médiée par les capacités recognitionnelles d'un sujet ${ }^{2}$. Cette relation est d'autant plus riche que ces capacités le sont aussi :

(2) Si $X \subset X^{\prime}$, alors $S F_{X} \subset S F_{X}$ '

Pour celui qui est capable de reconnaître que la température a augmenté, non seulement en constatant la hausse du mercure dans le thermomètre, mais en appréciant des événements comme l'infime dilatation d'une barre de métal et en rapportant ces phénomènes à leur origine, toutes les conséquences objectives de la montée de la température tendent à indiquer cette hausse. Suivant cette explication, un être doté de capacités recognitionnelles asymptotiquement vastes reconnaîtrait la vérité d'une proposition à n'importe laquelle de ses conséquences objectives, et acquerrait la conviction de cette vérité au hasard de la première de ces conséquences qui lui serait évidente. En d'autres termes, plus les pouvoirs de perception, d'inférence et de discrimination des sujets sont larges, plus le nexus des conséquences subjectives qu'ils sont capables d'établir entre propositions est riche, et plus la manière dont ils se convainquent de la vérité des propositions est susceptible de varier. Cette variation sans loi, qui va de pair avec la richesse et la diversité des possibilités d'expérience et d'inférence, est à l'exact opposé de la rigidité et de l'invariance du réseau des relations de conséquence objective entre propositions : jamais la variété des motifs de la conviction n'est aussi éloignée de la fixité des fondements objectifs que lorsque l'on est en présence de ressources cognitives indéfiniment riches et prolixes.

\section{2.}

La relation de conséquence objective - la fondation - est une relation "ontologique» dont la réalisation est parfaitement indépendante du point de savoir si elle a été ou non contemplée ou reconnue, et qui serait ce qu'elle

2. Bolzano explique la différence en ayant recours à la distinction qu'il établit d'entrée de jeu dans la Wissenschaftslehre entre propositions objectives ou en soi et propositions subjectives — aussi appelées jugements et, lorsqu'elles sont vraies, connaissances - c'est-à-dire l'épisode mental dans lequel la proposition objective est saisie (aufgefasst) par le sujet. Selon Bolzano, tandis que la relation de conséquence objective est entre des propositions objectives, la conséquence subjective est une relation est entre des connaissances (Bolzano, 1837, \$198 note, vol. 2, p. 341). 
est même si sa contemplation n'avait de vertu explicative pour personne. En d'autres termes, le fait que la reconnaissance de la vérité d'une certaine proposition rende intelligible la vérité de ses conséquences objectives est un simple sous-produit qui n'a pas à figurer dans la définition de la notion de conséquence objective elle-même : cette relation ne saurait être infiltrée de connotations épistémiques comme celles relatives à la «valeur explicative» pour un sujet, et des conséquences de ce genre, s'il en existe, sont l'apanage d'éventuelles présentations linguistiques du réseau objectif qu'elle détermine. Quid, cependant, de la réalité de ces conséquences?

Bolzano soutient que l'ordre idéal, strictement scientifique (streng wissenschaftlich) d'une science doit refléter la connexion objective des vérités qui lui sont propres, c'est-à-dire se conformer à l'ordre des conséquences objectives entre les propositions concernées. Ses interprètes ${ }^{3}$ insistent fréquemment sur la vertu explicative d'une présentation de ce type, qui établirait non seulement que les propositions en question sont vraies, mais pourquoi elles le sont. De façon générale, les explicativistes soutiennent que la présentation strictement scientifique au sens de Bolzano accomplit plus que ne le font ses rivales, supposées n'établir que la vérité des propositions auxquelles elles concluent : les présentations conformes à l'ordre de la conséquence objective établiraient également la vérité des propositions en jeu, mais elles le feraient, quant à elles, en montrant en outre le fondement de cette vérité. Nous prétendons que cette interprétation est, en général, erronée, que la reconnaissance de la vérité des propositions établies n'est pas une conséquence que l'on puisse attendre de la seule exposition «strictement scientifique " que Bolzano appelait de ses vœux, en d'autres termes que cette exposition ne fait pas plus que ses rivales, mais autre chose.

La plupart des explicativistes entendent se placer sur un terrain particulier qui, pour des raisons sur lesquelles nous reviendrons, leur est éminemment favorable, à savoir celui des propositions mathématiques. Aussi allons-nous plutôt commencer par la question générale, qui inclut notamment les principes de l'exposition «strictement scientifique » des propositions empiriques. À maintes reprises ${ }^{4}$, Bolzano fait référence, pour expliquer sa propre distinction entre la conséquence subjective et la conséquence objective, à la fameuse séparation proposée par Aristote dans les Seconds Analytiques entre les preuves qui n'établissent que la réalité (le otı) de la chose prouvée

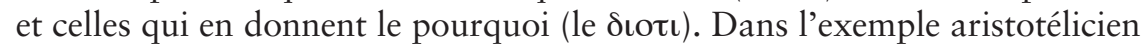
qui illustre la distinction ${ }^{5}$, deux manières d'établir des vérités astronomiques sont opposées. La proximité des planètes peut être établie en invoquant leur

3. Kitcher, P., «Bolzano's Ideal of Algebraic Analysis ", Studies in History and Philosophy of Science, 6, p. 237s., et Mancosu, P., "On Mathematical Explanation ", dans The Growth of Mathematical Knowledge, dir. E. Grosholz \& H. Breger, Dordrecht, Kluwer, 2000, p. 114s.

4. Par exemple, Bolzano, 1837, $\$ 198$ note, vol. 2, p. 341.

5. Aristote, Seconds Analytiques., I, 13 (trad. fr. Jean Tricot, Paris, Vrin, 2000). 
absence de scintillement, ce qui est un raisonnement conduisant à un simple fait, puisque la proximité des planètes n'a pas leur absence de scintillement pour fondement; mais on peut également présenter les choses de manière à conclure de la proximité des planètes à leur absence de scintillement, ce qui est alors conclure du fondement à la conséquence. Cet exemple illustre bien le malentendu sur lequel repose l'explicativisme. Pour ce dernier, la connaissance du pourquoi d'un fait nous donne plus que la simple connaissance de ce fait, puisqu'elle nous donne ce fait et son fondement : il serait inconcevable qu'on nous donne l'explication d'un fait qui ne se produirait précisé-

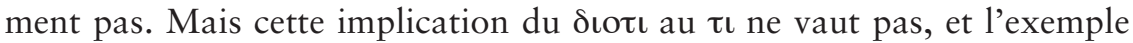
aristotélicien montre pourquoi : ce dont la réalité est affirmée (la proximité des planètes) n'est justement pas ce dont le fondement est établi (leur absence de scintillement). Il n'y a pas un fait que l'on pourrait indifféremment présenter en montrant qu'il a lieu et en expliquant pourquoi il se produit : il y a deux faits dont l'un est fondement de l'autre, et la réalité de celui qui est établi est connue par sa conséquence et non pas par son fondement.

L'explicativiste soutient qu'un certain type de présentation est capable à la fois de nous convaincre de la réalité d'un fait et de nous indiquer la nature de son fondement : $\phi$, et $\phi$ parce que $\psi$. Bien plus, il soutient que l'indication du fondement est, dans cette présentation, non seulement concomitante de la reconnaissance de la réalité de la conséquence, mais qu'elle est elle-même la raison — le fondement subjectif — de cette reconnaissance : $\phi$, parce que $\phi$ parce que $\psi$. Naturellement, la conception bolzanienne de la conséquence objective autorise largement cette interprétation : la relation de conséquence objective ne vaut qu'entre propositions vraies, en sorte que celui qui a reconnu que $\phi$ est une conséquence objective de $\psi$ ne peut manquer d'avoir reconnu que $\phi$ et que $\psi$. Néanmoins, l'exemple aristotélicien montre que cette interprétation est, en général, inadéquate. Le jugement selon lequel les planètes ne scintillent pas, parce qu'elles sont proches, est la conjonction $\mathrm{du}$ jugement selon lequel les planètes sont proches, et du jugement selon lequel l'éloignement d'un corps céleste est une condition suffisante pour qu'il scintille. Dès lors, de deux choses l'une. Ou bien la "présentation » que l'explicativiste a en vue est la présentation de ces deux propositions conjuguées, et l'on voit que la reconnaissance du fait est déjà requise pour que la reconnaissance du fondement soit obtenue. Ou bien cette "présentation » n'inclut que la proposition conditionnelle qui concerne la nécessité du lien entre deux événements hypothétiques (S'il n'y a pas scintillement, alors il y a proximité), et il est alors exclu que l'on puisse dériver de cette proposition conditionnelle la reconnaissance d'un fait. En résumé, ou bien la connaissance nue du fait est requise pour établir son fondement, ou bien l'établissement du fondement est insuffisant pour attester la réalité de la conséquence. Dans les deux cas, il est erroné de tenir la connaissance du pourquoi pour un mode de la connaissance du que : la seconde connaissance ne découle de la première que si elle y a été expressément agrégée, et donc si elle provient d'une autre 
source qu'elle. L'explicativiste soutient que, dans la présentation qui suit l'ordre de la connaissance objective, la reconnaissance de ce qui entraîne la chose entraîne la reconnaissance de la chose. C'est admettre une variante de la conception «détectrice» de la preuve qui est en général intenable.

\section{3.}

Aristote établit exactement la condition supplémentaire qui serait requise pour que le phénomène visé par l'explicativiste se produise effectivement. Il faudrait, dit-il ${ }^{6}$, que l'élément qui est le fondement soit également celui qui est le mieux connu. À cette condition seulement, la connaissance du pourquoi a pour conséquence la connaissance du que : s'il est connu que la réalisation de $\phi$ entraîne celle de $\psi$, et si $\phi$ est connu, alors $\psi$ l'est également, et il l'est « par son fondement ». Cette condition supplémentaire n'est évidemment satisfaite, ni dans l'exemple bolzanien de la hausse de la température, ni dans l'exemple aristotélicien de la proximité des planètes, puisque la conséquence y est, à chaque fois, mieux connue que le fondement, et que la connaissance du fondement découle donc de la connaissance de la conséquence et non l'inverse : comme l'écrit Aristote, "dans les cas où le terme plus connu est celui qui n'est pas cause, c'est le fait qui est démontré, et non le pourquoi » ${ }^{7}$.

Convenons d'appeler désormais connaissance par le fondement (par opposition à la connaissance $d u$ fondement, qui est la simple connaissance d'un lien conditionnel entre deux propositions) cette connaissance qui établit la conséquence par le biais de son fondement mieux connu que lui. Quelles sont les caractéristiques de cette connaissance, et à quelles conditions est-elle possible?

Il est clair que, lorsque cette connaissance apparaît, la relation particulière de conséquence subjective qui est obtenue est une partie de la relation de conséquence objective, et non pas de sa converse :

(3) $S F^{*} \subset O F$

Cette relation particulière de conséquence subjective ( « $\left\{\phi_{1}, \ldots, \phi n\right\} S F^{*}$ $\phi »$ signifie « $\phi$ est connue par ses fondements $\left.\phi_{1}, \ldots, \phi_{n} »\right)$ soulève, pour l'essentiel, deux questions. Il y a, d'une part, celle de savoir si la relation $S F^{*}$ est, comme la relation $S F$ de conséquence subjective "ordinaire ", dépendante des capacités recognitionnelles des sujets. La réponse à cette question est négative, puisque $S F^{*}$ n'est rien d'autre que la présentation linguistique de la relation de conséquence objective, qui est, quant à elle, "intrinsèque » et bien déterminée. À un détail près, sur lequel nous reviendrons, cette relation

6. Aristote, Seconds Analytiques, 78 a 27-28; L'usage que fait Aristote de «cause » correspond à notre usage de «fondement ». Dans la suite de l'article, pour les fins de la clarté, de nous privilégierons ce dernier terme.

7. Aristote, Seconds Analytiques, 78 b 11. 
est donc unique. Il y a, d'autre part, la question de savoir si cette connaissance par les fondements est en général possible, même dans le cas où la condition d'Aristote est satisfaite. Nous essaierons d'argumenter qu'elle ne l'est pas, et que l'interprétation explicativiste de Bolzano est donc dénuée de pertinence.

\section{4.}

S'agissant de la question de l'unicité, il est clair que la connaissance «par les fondements " n'est pas sujette aux mêmes variations que la connaissance ordinaire (dans l'exemple météorologique pris par Bolzano, la hausse de la température peut être conclue de n'importe laquelle de ses conséquences perçues, dont l'étendue varie sans règle en fonction des capacités de discrimination des sujets). Ici, cette variation en aval, du côté des conséquences, n’a évidemment pas lieu. Mais elle est éventuellement compensée par une variation en amont, du côté des fondements de la proposition connue. C'est là un point dont Aristote s'explique dans le même chapitre des Seconds Analytiques, lorsqu'il entend disqualifier, comme candidates au titre de connaissances par les fon-

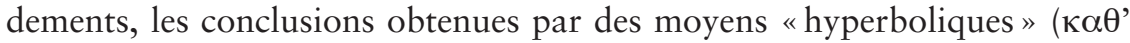
$v \pi \varepsilon \rho \beta 0 \lambda \eta)$, c'est-à-dire inférées à partir de propositions exagérément éloignées dans l'ordre de la fondation. On est dans ce cas de figure, dit Aristote, lorsque l'on conclut qu'il n'y a pas de joueurs de flûte chez les Scythes parce qu'on ne cultive pas la vigne dans leur pays, alors qu'il suffirait, pour conclure à cette absence, d'invoquer le fait que les Scythes ne sont jamais suffisamment ivres pour se livrer à ce passe-temps. Au contraire, la connaissance par le fondement doit être entendue au sens du fondement "prochain ", et non pas du fondement «éloigné ». C'est le sens dans lequel on comprend que les murs ne respirent pas en réalisant qu'ils n'ont pas de poumons, et non pas en se souvenant, fondement trop éloigné, que ce ne sont pas des animaux ${ }^{8}$. De la même façon, Bolzano traite la relation de conséquence objective comme une relation dérivée, à savoir comme la clôture transitive d'une relation plus simple reliant un ensemble de propositions à celles qui en sont des conséquences objectives immédiates : $\phi$ est une conséquence objective de $\phi_{1}, \ldots, \phi_{n}$ exactement dans le cas où il existe une "chaîne" de conséquences immédiates partant de $\phi_{1}, \ldots, \phi_{n}$ et se terminant par $\phi$. Dans ces conditions, il existe, pour chaque proposition vraie, une arborescence objective unique qui la relie de manière ordonnée aux propositions qui la fondent et dont elle est une conséquence objective. Connaître une proposition vraie par son fondement consiste alors à saisir les propositions vraies dont elle est une conséquence objective immédiate, ce qui exclut toute variance individuelle dans la détermination des fondements effectivement mis en avant dans l'élaboration de cette connaissance.

La seule indétermination résiduelle provient alors du fait que la présentation linguistique linéaire de l'arborescence des fondements doit composer

8. Ibid., 78 b 12 s. 
avec l'absence d'un ordre total entre ces derniers. En effet, cette arborescence peut avoir, par exemple, la forme suivante, où $\phi_{11}=\phi_{1}, \ldots$, et $\phi q p_{\mathrm{q}}=\phi_{\mathrm{n}}$

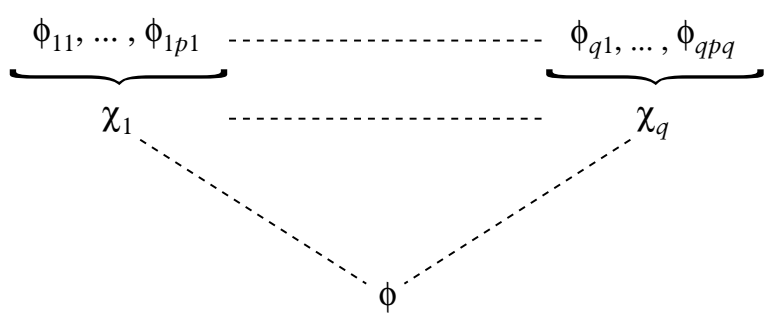

Dans cet exemple, $\chi_{1}$ et $\chi_{q}$ sont respectivement conséquences immédiates de $\phi_{11}, \ldots, \phi_{1 p 1}$ et de $\phi_{q 1}, \ldots, \phi_{q p q}$, mais aucune des deux propositions ne précède l'autre dans l'arborescence. La "chaîne» des relations de conséquence doit donc s'entendre à une permutation près des propositions incomparables qui y figurent : des deux propositions Caius est au forum et Scipion est au forum, auxquelles la proposition Caius et Scipion sont au forum doit sa vérité, aucune ne peut être considérée comme précédant l'autre dans l'arbre des conséquences objectives immédiates ${ }^{9}$. Autrement dit, c'est bien d'un ensemble non structuré de propositions qu'une proposition peut être considérée comme conséquence immédiate, et à cet égard une forme minimale et vénielle de variation interindividuelle peut se manifester dans le processus, nécessairement diachronique, de la reconnaissance «fondationnelle » des propositions vraies.

\section{5 .}

L'explicativiste entend donner une interprétation épistémique à l'idée bolzanienne d'une structure objective des liens de fondation immédiate entre propositions vraies. Selon lui, la reconnaissance de la vérité des propositions selon cette structure hiérarchique possède une vertu dont tout autre type de reconnaissance est dénué, puisqu'elle seule permet de saisir les propositions vraies comme il convient, c'est-à-dire par le biais des propositions auxquelles elles doivent leur vérité. Pour que cette saisie "canonique» soit possible, quatre propriétés au moins devraient être satisfaites par la structure objective en question :

(i) En vertu de l'argument aristotélicien développé plus haut, les propositions auxquelles les propositions examinées doivent leur vérité devraient être au moins aussi faciles à reconnaître que ces dernières

(ii) La structure d'ensemble des liens qui relient la proposition examinée à celles dont elle est conséquence objective immédiate devrait être finîment maîtrisable (principe de finitude «horizontale»)

9. Bolzano, 1837, $\$ 211$, vol. 2, p. 369. 
(iii) L'arborescence des liens qui relient régressivement, de proche en proche, la proposition examinée à la totalité de ses antécédents doit être bien fondée (principe de finitude "verticale»)

(iv) Chacun des liens de conséquence objective entre la proposition examinée et les propositions qui sont à la source de sa vérité doit pouvoir être individuellement reconnu comme tel.

5.1.

La première condition, dont la satisfaction problématique constitue une objection à l'explicativisme en général, a déjà été discutée plus haut : l'interprétation explicativiste est au mieux applicable aux domaines où ce réquisit est satisfait.

5.2 .

La condition de finitude horizontale pourrait ne pas être satisfaite dans le cas, par exemple, des propositions universelles relatives à un domaine infini d'objets. Dans une interprétation du quantificateur universel comme conjonction généralisée, la proposition selon laquelle, par exemple, tous les triangles équilatéraux sont équiangulaires, pourrait devoir immédiatement sa vérité à la collection de toutes les propositions selon lesquelles tel ou tel triangle équilatéral est équiangulaire, collection de propositions qu'une créature finie serait bien en peine de reconnaître individuellement comme vraies dans leur totalité. Bolzano récuse cette interprétation, et met en avant une analyse des propositions qui est de nature à écarter cette indominable infinité. Les propositions sont conçues par lui comme des entités complexes dont les parties peuvent ne pas être ellesmêmes des propositions, mais des «représentations en soi » (Vorstellungen an sich $)^{10}$, une proposition étant typiquement constituée du lien entre une représentation-sujet (Subjectsvorstellung) et une représentation-propriété (Beschaffenheitsvorstellung) qu'elle lui subordonne. Dans ces conditions, l'usage du quantificateur «tous les ...» dans l'expression verbale de la proposition considérée, loin d'être l'application d'un constituant authentique, n'est qu'une façon, d'ailleurs explétive, de rappeler que la représentation-sujet, qui est singulière, doit être considérée sans aucune restriction : c'est du triangle équilatéral en toute généralité dont il est question. Dire que la représentation triangle équilatéral est singulière, c'est affirmer qu'elle n'a pas à être conçue comme distribuée sur la collection infinie des triangles équilatéraux individuels qui sont dans son extension et que c'est elle, dans son intégralité, qui constitue le «thème» de la proposition. Ce n'est pas dire, toutefois, que cette représentation soit en elle-même simple ou indivise : cette représentation est complexe, en tant qu'elle possède pour parties d'autres représentations : triangle, qui, a, et équilatéralité, ainsi que les représentations qui, le cas échéant, sont les parties

10. Ibid., $\$ 48$, vol. 1, p. 216 . 
de ces dernières, celles qui sont les parties de ces parties, etc ${ }^{11}$. Il se pourrait donc qu'une infinitude "horizontale» se réintroduise par ce biais, une proposition vraie étant conséquence immédiate d'une collection infinie de propositions contenant des représentations diverses.

Pour une certaine classe de propositions, à savoir les propositions "purement conceptuelles », (reine Begriffssätze), par opposition aux propositions empiriques, Bolzano montre que cette situation ne peut pas se produire. L'argument tient en trois points ${ }^{12}$ :

(i) Une proposition purement conceptuelle ne peut être conséquence immédiate que de propositions de même nature qu'elle (ceci a pour contrepartie épistémique que la vérité d'une proposition purement conceptuelle doit pouvoir être reconnue par le seul exercice de la réflexion conceptuelle).

(ii) La complexité conceptuelle d'une proposition, c'est-à-dire le nombre de concepts qu'elle contient, ne peut excéder la complexité conceptuelle des propositions qui lui succèdent immédiatement dans l'arborescence des conséquences objectives.

(iii) Le nombre des concepts simples est fini, aussi bien que les modalités de leur agencement dans une proposition.

Par (i), les antécédents immédiats d'une proposition purement conceptuelle sont tous des propositions purement conceptuelles, qui ne se distinguent donc les unes des autres que par les concepts simples qu'elles contiennent et par les modes d'agencement de ces concepts. Par (ii), le nombre de concepts contenus dans ces antécédents est inférieur à celui, disons $n$, des concepts contenus dans la proposition examinée. Par (iii), cette proposition ne peut avoir une infinité d'antécédents immédiats distincts, puisque chacun de ceux-ci est complètement déterminé par le choix d'au plus $n$ concepts à choisir dans un ensemble fini et à ordonner selon un nombre fini de modalités.

\section{$5 \cdot 3$.}

Est-il également garanti, dans le domaine des vérités purement conceptuelles, que la régression d'une proposition aux propositions dont elle est conséquence immédiate a une fin ? De même que certaines propositions sont conséquences immédiates d'une infinité d'autres, comme celles qui, en vertu de leur caractère empirique, requièrent pour être établies une collection sans fin d'intuitions (ceci est $\phi$, ceci est également $\phi$, etc) ou tirent leur vérité d'elles,

11. Ibid., $\$ 59$, vol. 1, p. 257s. La conception bolzanienne de la composition des représentations sous-tend une syntaxe logique au sein de laquelle deux règles récursives sont particulièrement importantes : l'attribution (par exemple, "triangle qui a l'équilatéralité ", "Figure qui a trois angles, qui a l'équilatéralité ", etc.) et la conjonction ( "triangle qui est isocèle, droit, etc...») Cette manière de concevoir l'articulation du contenu de la représentation, en termes syntaxiques, constituait alors une nouveauté en logique.

12. Ibid., $\$ 221$, vol. 2, p. 384s. 
il se pourrait en effet que l'arborescence d'autres propositions contienne des branches infinies ${ }^{13}$. L'argument précédent, qui repose sur la finitude du nombre des concepts et du nombre de leurs modalités d'arrangement, est ici insuffisant pour exclure cette possibilité. Il assure seulement qu'une vérité conceptuelle ne peut posséder une infinité d'antécédents immédiats distincts, la finitude du nombre de ces antécédents immédiats tout court provenant alors du fait que leur collection est traitée, ontologiquement, comme un ensemble non structuré dans lequel les éléments de type identique sont identifiés, plutôt que comme ce que les logiciens substructuralistes nomment un "panier ", et où le nombre des occurrences serait pertinent. Il est impossible d'invoquer ici une particularité de ce type, justement parce que l'arborescence bolzanienne est verticalement structurée, alors qu'elle ne l'est pas horizontalement. Qu'il suffise de mentionner le fragment suivant d'une dérivation, évidemment bâtie pour les besoins de la cause, de la proposition selon laquelle 2 est pair :

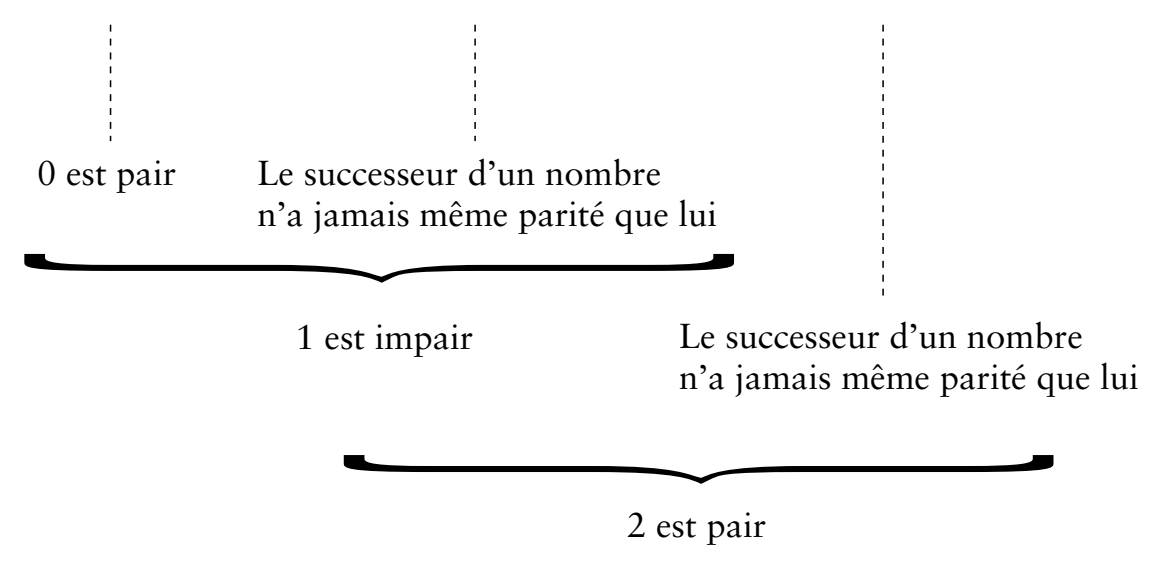

Néanmoins, l'apparition de plusieurs exemplaires de la même proposition dans l'arborescence «causale » d'une proposition purement conceptuelle est tout à fait naturelle, et elle n'est pas de nature à engendrer ce qu'il fallait précisément éviter, à savoir l'existence d'une branche infinie dans cette arborescence. En effet, les propositions identiques n'apparaissent jamais sur le même chemin, et l'impossibilité de tout «cycle» de ce type est, en fait, garantie par le principe bolzanien selon lequel la relation de conséquence objective est irréflexive. À cet égard, il est établi que la connaissance "par les fondements " est assurée de parvenir, régressivement, à des propositions "primitives » qui ne sont elles-mêmes conséquences d'aucune autre, ce qu'il fallait obtenir. 


\section{4 .}

S'agissant enfin de la possibilité de reconnaître les liens de dépendance objective immédiate entre propositions, il est généralement admis qu'elle est la contrepartie naturelle de l'indécomposabilité de ces liens. L'argument est que, si le lien de dépendance objective de $\phi$ par rapport à une autre proposition $\psi$ était irrémédiablement opaque, ceci signifierait qu'une certaine information propositionnelle «médiane» manque d'être aperçue, et que, par conséquent, la dépendance de la vérité de $\phi$ par rapport à celle de $\psi$ n'est pas à proprement parler immédiate. Autrement dit, l'intelligibilité requise est garantie par la minimalité de la relation de conséquence objective immédiate : il ne saurait $\mathrm{y}$ avoir aucune espèce de doute insurmontable sur la nature du lien entre une proposition et chacune de celles dont elle tire sa vérité, puisque si une raison manque d'être aperçue, c'est qu'une autre s'intercale entre elle et ce dont il s'agit de rendre raison. La contrepartie épistémique de la structure objective détaillée (id est déterminée jusque dans les liens objectivement indécomposables qui la constituent) de la réalité est l'existence de pas inférentiels minimaux dont la correction, pour cette raison, ne doit faire de doute pour aucune créature rationnelle. À l'inverse des preuves qui ne sont destinées à s'assurer que de la vérité de ce qu'elles démontrent, et où il suffit que les règles de passage d'un énoncé à l'autre transmettent régulièrement à leur conclusion la vérité éventuelle de leurs prémisses, une preuve qui transcrit les raisons immédiates de cette vérité ne doit admettre que des transitions beaucoup plus contraintes. Ainsi que l'écrit Frege dans un contexte voisin,

La plupart du temps, on se contente du fait que chaque pas de la preuve est évidemment correct, ce qui est légitime si l'on n'est préoccupé que de la vérité de la proposition à prouver. Mais, lorsqu'il s'agit d'obtenir quelque aperçu sur la nature de cette évidence, ce procédé ne suffit pas, et nous devons, au contraire, expliciter toutes les étapes intermédiaires, afin de laisser tomber sur elles la pleine lumière de la conscience. D'ordinaire, les mathématiciens ne s'occupent que du contenu de la proposition, et du fait qu'elle est prouvée. Ici [dans les Grundgesetze], ce qui est nouveau n'est pas le contenu de la proposition, mais la manière dont la preuve est conduite, et les fondements sur lesquels elle repose $^{14}$.

\section{6.}

Avec les propositions "purement conceptuelles », et notamment les propositions mathématiques, l'explicativiste est donc sur un terrain éminemment favorable pour justifier sa thèse favorite : la présentation détaillée de l'arborescence fondationnelle des propositions vraies est de nature à nous faire reconnaître la vérité de ces propositions en nous indiquant en outre la raison de leur vérité, puisque cette arborescence nous montre de façon indiscutablement intelligible

14. Frege, Gottlob, Grundgesetze der Arithmetik, vol. 1, 1893, réimpr. Hildesheim, Georg Olms, 1966, p. VIII. 
leurs liens individuels avec les propositions auxquelles elles doivent leur vérité, que ces propositions-sources sont au moins aussi claires qu'elles, et que l'ensemble de ces liens possède les deux propriétés de finitude requises pour les réputer maîtrisables.

Nous ne disputerons pas ce qui précède, et concèderons sans autre examen les prémisses parfois controversées sur lesquelles reposent les propriétés cruciales qui viennent d'être énumérées, comme celle qui est relative à la finitude de l'ensemble des concepts simples. Bien plus, l'un des arguments d' "accessibilité » en question, celui qui est relatif à la finitude "verticale » des arborescences bolzaniennes, peut être renforcé de la manière suivante. Pour établir cette finitude, on a eu recours à l'argument selon lequel le nombre des concepts figurant dans les antécédents immédiats (et donc, de proche en proche, dans les antécédents tout court) d'une proposition conceptuelle donnée ne peut excéder celui des concepts qui figurent dans la proposition en question :

Si nous pensons réunies dans une collection toutes les vérités conceptuelles pures dont la complexité ne dépasse pas une certaine limite (par exemple toutes les vérités qui ne renferment pas plus de cent parties simples), il s'ensuit de ce qui précède que chaque vérité qui y figure et qui n'est pas primitive trouve dans cette provision de vérités non seulement sa raison prochaine, mais aussi toutes ses raisons éloignées ${ }^{15}$.

Or, c'est, après tout, l'ensemble des concepts simples qui figure dans ces antécédents, immédiats ou lointains, qui est lui-même inclus dans l'ensemble des concepts figurant dans la proposition fondée :

Si une proposition donnée ne consiste qu'en concepts, comme, par exemple, la proposition selon laquelle la vertu mérite respect, ou selon laquelle les deux côtés d'un triangle, pris ensemble, sont toujours plus longs que le troisième côté, etc., alors la vérité ou la fausseté de cette proposition ne dépend que des propriétés de ces concepts; et donc ce qui est requis, au moins dans la plupart des cas, pour nous convaincre de sa vérité, ce n'est rien de plus que de considérer avec attention les concepts qui y sont contenus. Ainsi, il est possible d'apprendre que la vertu mérite respect, à la seule condition de posséder les concepts de vertu et de respect (...). On apprend les vérités de ce type (les vérités purement conceptuelles) en vertu du fait que l'on connaît les concepts qu'elles contiennent. La chose est différente en ce qui concerne les jugements qui contiennent des intuitions (...). Pour ces propositions-là, on peut dire que leur vérité ne dépend pas seulement des représentations que l'on a, mais aussi des propriétés des objets extérieurs dont ce sont les représentations ${ }^{16}$.

En conséquence, ce n'est pas seulement que la connaissance canonique des vérités conceptuelles ne requiert aucune intuition, et qu'elle ne mobilise donc aucune des expériences singulières de ceci ou de cela que nous pourrions

15. Bolzano, 1837, $\$ 221$, vol. 2, p. 386 .

16. Ibid., $\mathbb{S} 42$, vol. 1 , p. 181. 
avoir, mais, aussi bien, ne pas avoir, ce n'est pas seulement, non plus, que la réflexion purement conceptuelle qu'elle exige des sujets ne suppose de leur part que la maîtrise d'un nombre fini de concepts simples, c'est que celui qui est capable de simplement énoncer ces vérités de ce genre est, eo ipso, en possession du répertoire conceptuel requis exigé pour en saisir la vérité. À quoi tiendrait alors, dans ce tableau sans ombres, la faiblesse de l'explicativisme?

Le programme de Bolzano vise à remplacer les preuves quelconques, les Gewissmachungen, celles qui n'entraînent que la conviction en la vérité de la proposition qu'elles prouvent, par des preuves fondamentales (Begründungen) qui procèdent en indiquant à chaque étape les propositions auxquelles les conclusions sont immédiatement redevables de leur vérité ${ }^{17}$. De ce programme, on ne connaît que quelques réalisations illustres, comme celle qui consiste à établir le théorème des valeurs intermédiaires de manière "pure ", c'est-à-dire sans introduire de concepts géométriques ou cinématiques réputés étrangers à la proposition prouvée. Néanmoins, nous en connaissons assez, depuis les recherches de Gentzen ${ }^{18}$ pour savoir que ce programme, s'il est en général réalisable, comporte certains inconvénients auxquels l'explicativiste ne semble pas prêter une attention suffisante.

Pour l'essentiel, la transformation d'une preuve « ordinaire» en preuve bolzanienne consiste à éliminer les configurations inférentielles par lesquelles s'introduisent dans cette preuve des concepts étrangers à la proposition prouvée. Ainsi, une configuration comme

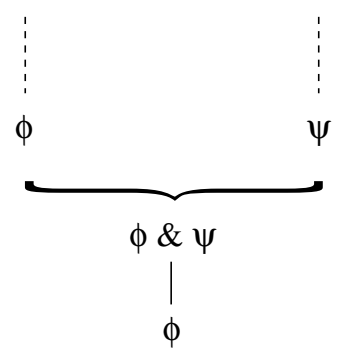

sera remplacée par la configuration réduite à « $\phi$ », puisque $\psi$ et ses antécédents n'ont certainement pas qualité à figurer parmi les propositions auxquelles $\phi$ doit sa vérité. On peut démontrer que l'ordre dans lequel sont effectués ces remplacements ou ces effacements est sans importance : le processus possède la propriété dite de «Church-Rosser », selon laquelle son résultat est une preuve «bolzanienne» unique et bien définie quelle que soit la manière dont on s'y prend pour l'obtenir.

17. Ibid., $\$ 525$, vol. 4, p. 261.

18. Pour une mise en rapport des idées de Bolzano avec la théorie gentzénienne de la démonstration, cf. par exemple K. Schröter, "Theorie des Logischen Schliessens ", I et II, Zeitschrift für mathematischen Logik und Grundlagen der Mathematik, I-1955, p. 37-86 et IV1958, p. 10-65, ainsi que J. Berg, Bolzano’s Logic, Stockholm, Almqvist \& Wiksell, 1962. 
A vrai dire, il serait difficile d'imaginer pourquoi le raisonnement par lequel nous en viendrions à nous convaincre de la vérité de $\phi$ prendrait la forme précédente : des considérations élémentaires d'économie cognitive nous conduiraient certainement à nous arrêter après la première occurrence de " $\phi$ ", plutôt qu'à agréger une preuve de $\psi$ à la preuve déjà obtenue pour $\phi$ avant d'en conclure à nouveau $\phi$. Tout autre est le cas du modus ponens, c'est-à-dire de la configuration ( «coupures») :

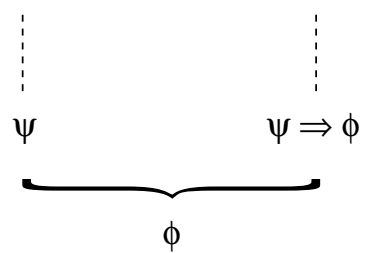

Ici, aucune redondance visible n'a plus cours. J'ai peut-être pris de la vérité de $\psi \Rightarrow \phi$ une connaissance de seconde main, la tenant de mon ami Wisdom, qui de son côté en possédait, mais peut-être pas, une preuve canonique. Quoi qu'il en soit, je ne déballe pas sa preuve, et la prends pour lemme, ayant assez affaire, de mon propre côté, pour établir $\psi$. J'aurais pu, il est vrai, ne pas connaître Wisdom, ou ne pas lui avoir inspiré suffisamment confiance pour qu'il me livre son résultat. Ceci est de l'ordre des expériences que j'aurais pu ne pas avoir, tout comme j'aurais pu ne pas avoir l'expérience empirique de ceci ou de cela. Une créature réduite à des pouvoirs recognitionnels minimaux n'aurait pas connu Wisdom, et un sujet doté de pouvoirs moins modestes, mais décidé à s'abstenir du bénéfice de tout exercice particulier de ces dispositions, n'aurait pas profité de ses avis. Moins humble, ou moins puritain, j'ai cédé à ces facilités, et j'ai désormais une preuve qui me rend certain de la vérité de $\phi$, mais qui ne m'en donne pas le fondement ${ }^{19}$.

Veritatis amicus, sed veritatis causae magis amicus? Arrivé à ce point, supposons que nous nous rendions aux objurgations de l'explicativiste, qui nous représente l'avantage de transformer la preuve impure à laquelle je suis parvenu en une démonstration qui nous donnera, nous assure-t-il, l'avantage supplémentaire de savoir pourquoi son résultat est vrai. Nous avons en effet l'assurance, si nous procédons suivant la méthode préconisée par Gentzen pour éliminer les unes après les autres les configurations ( "coupures») du type précédent, de parvenir à une preuve bolzanienne bona fide, où il n'est de proposition qui ne soit effectivement dérivée de ses fondements propositionnels les plus immédiats, et où il ne se rencontre de concept qui ne soit effec-

19. Bolzano n'ignore pas que des contraintes pragmatiques surviennent dans l'exercice effectif de la démonstration et il en discute de manière détaillée (Cf. Bolzano, 1837, \$512, vol. 4, p. 237s.). Mais même dans le contexte pragmatique, il défend l'idée que l'on doit, aussi souvent que cela est possible, "amener le lecteur à une claire conscience de l'ensemble des causes objectives (Grund) sur lesquelles repose une preuve.» (Ibid., $\$ 517$, vol. 4, p. 248). 
tivement contenu dans le résultat (propriété de la «sous-formule»). Mais quel est au juste l'objet résultant, à supposer que nous parvenions à le construire? Si la longueur de ma Gewissmachung initiale est $d$, et si la complexité de $\phi$ est $n$, alors la longueur de la chose obtenue est donnée par une tour d'exponentielles :

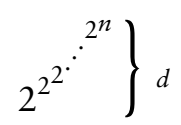

Il se trouve peut-être des explicativistes pour estimer que nous n'avons rien perdu au change, en troquant pour une preuve par excellence la démonstration impure qui était le motif de ma conviction rationnelle. Peut-être certains d'entre eux soutiendraient-ils qu'il n'y a à cet égard, entre une preuve impure qui tient en deux pages et une preuve par les fondements dont le nombre de caractères excède celui des nanosecondes écoulées depuis le BigBang ${ }^{20}$, aucune différence qui mérite que l'on s'y attarde, que c'est bien assez, pour la théorie de la connaissance, que de prendre garde aux impossibilités de principe, et que s'il fallait en outre se préoccuper des incapacités «médicales » qui s'y surajoutent, la philosophie tout entière se perdrait en détails. Pour notre part, nous voyons dans une défense de ce genre une méconnaissance patente de la différence entre ontologie et épistémologie : les propriétés ontologiques, comme la fondation, sont conservées par passage d'une relation à sa clôture transitive, alors que les propriétés épistémologiques, comme l'intelligibilité, ne le sont pas.

20. G. Boolos, «Don't Eliminate Cut ", dans Logic, Logic, and Logic, Harvard U. Press, 1998, p. 365-369. 